\title{
Escala diagramática para avaliação da severidade da cercosporiose em caupi
}

\author{
Diagrammatic scale for assessment of Cercospora leaf spot severity in cowpea
}

\author{
Igor Corrêa Lima Albert ${ }^{\mathrm{I}}$ Marissônia de Araújo Noronha' ${ }^{\mathrm{II}}$ Ricardo Brainer Martins ${ }^{\mathrm{III}}$ \\ Sami Jorge Michereff ${ }^{*}$
}

\begin{abstract}
A cercosporiose, causada por Cercospora RESUMO cannescens e Pseudocercospora cruenta, é uma importante doença do caupi (Vigna unguiculata) no Brasil. Devido à inexistência de métodos padronizados para quantificação dessa doença, foi desenvolvida uma escala diagramática com níveis de 1, 2, 4, 8, 16, 32, 64 e 82\% de área foliar lesionada. A escala diagramática foi validada por 10 avaliadores, usando 50 folíolos de caupi com diferentes níveis de severidade, mensurados previamente com o programa Assess ${ }^{\circledR}$. A acurácia, a precisão e a reprodutibilidade das estimativas de cada avaliador foi determinada por regressão linear simples entre a severidade real e a estimada, com $e$ sem o auxílio da escala. Com a escala, os avaliadores obtiveram melhores níveis de acurácia e precisão das estimativas, com os erros absolutos concentrando-se na faixa de $10 \%$. Os avaliadores apresentaram elevada repetibilidade (94\%) e reprodutibilidade ( $\geq 90 \%$ em $82,3 \%$ dos casos) das estimativas com a utilização da escala. Portanto, a escala diagramática proposta é adequada para avaliação da severidade da cercosporiose do caupi.
\end{abstract}

Palavras-chave: Vigna unguiculata, Cercospora canescens, Pseudocercospora cruenta, fitopatometria.

\section{ABSTRACT}

Cercospora leaf spot caused by Cercospora canescens and Pseudocercospora cruenta is an important disease of cowpea ([Vigna unguiculata) in Brazil. Due to the inexistence of standard methods for the assessment of this disease, a diagrammatic scale was developed with 1, 2, 4, 8, 16, 32, 64 and $82 \%$ of diseased leaf area. The diagrammatic scale was validated by 10 raters using 50 cowpea leaflets with different levels of severity previously measured by the software Assess ${ }^{\circledR}$. The accuracy, precision and reproducibility of the estimative of each rater were determined by simple linear regression between actual and estimated severity, with and without the use of the scale. With the scale raters obtained better levels of accuracy and precision, with absolute errors concentrating around $10 \%$. Raters showed high repeatability (94\%) and reproducibility ( $\geq 90 \%$ in $82.3 \%$ of the cases) of the estimates by using the scale. The proposed diagrammatic key is suitable for the evaluation of Cercospora leaf spot severity in cowpea.

Key words: Vigna unguiculata, Cercospora canescens, Pseudocercospora cruenta, phytopatometry.

A cercosporiose, causada por Cercospora canescens Ellis \& Martin e Pseudocercospora cruenta Sacc., é uma importante doença do caupi [Vigna unguiculata (L.) Walp.] no Brasil, podendo causar reduções superiores a $40 \%$ na produção de grãos. Os sintomas ocorrem nos folíolos, inicialmente como manchas irregularmente circulares, necróticas, secas e ligeiramente deprimidas. As lesões induzidas por $\boldsymbol{C}$. canescens são alaranjadas ou marrom-claras, enquanto de $\boldsymbol{P}$. cruenta são castanho-escuras ou avermelhadas. Com a evolução da doença, o centro das lesões tornase pardo-acinzentado e o bordo escurecido, com abundante esporulação na superfície adaxial das folhas. As lesões podem coalescer e formar grandes áreas necróticas, levando à queda prematura das folhas (PIORIBEIRO et al., 2005).

IDepartamento de Agronomia, Área de Fitossanidade, Universidade Federal Rural de Pernambuco (UFRPE). Av. Dom Manoel de

Medeiros, s/n, 52171-900, Recife, PE, Brasil. E-mail: sami@depa.ufrpe.br. *Autor para correspondência.

IILaboratório de Fitopatologia, Embrapa Meio-Norte, Teresina, PI, Brasil.

IIIPrograma de Pós-graduação em Fitopatologia, Universidade Federal de Viçosa (UFV), Viçosa, MG, Brasil. 
A severidade da cercosporiose do caupi tem sido avaliada principalmente com o auxílio de escalas descritivas de notas (EDEMA et al., 1997; COÊLHO \& COELHO, 1997) e contagem do número de lesões por folíolo (RIOS \& ZIMMERMANN, 1987). Como os procedimentos de quantificação de doenças devem ser de fácil e rápida utilização para uma ampla gama de condições, mas também propiciar resultados acurados, precisos e reprodutíveis (NUTTER Jr \& SCHULTZ, 1995), os métodos utilizados na quantificação da severidade da cercosporiose do caupi apresentam sérias limitações, principalmente a subjetividade das estimativas com escalas descritivas e o tempo demandado para contagem do número de lesões. Várias estratégias têm sido propostas para minimizar a subjetividade das estimativas de severidade, entre as quais se destacam as escalas diagramáticas (BERGAMIN FILHO \& AMORIM, 1996). Nesse contexto, este trabalho teve como objetivos desenvolver uma escala diagramática para avaliação da severidade da cercosporiose em caupi e analisar os níveis de acurácia, precisão e reprodutibilidade das estimativas geradas com sua utilização.

Para elaboração da escala diagramática, foram coletados 100 folíolos de caupi (cv. IPA-206) em plantios comerciais no município de Goiana - PE, com diferentes níveis de severidade da cercosporiose. Os folíolos foram digitalizados a 200 dpi e, com auxílio do programa Assess ${ }^{\circledR}$ (The American Phytopathological Society, St. Paul, MN, USA), foi determinada a porcentagem de área lesionada de cada folíolo, representando o grau de severidade da doença. Baseando-se na lei de Weber-Fechner de acuidade visual (HORSFALL \& COWLING, 1978), bem como no valor máximo de severidade da doença constatada nos folíolos coletados, na forma, na distribuição e na freqüência das lesões, foi confeccionada uma escala diagramática com oito níveis de severidade.

Na validação da escala diagramática, foram utilizados 50 folíolos de caupi com diferentes níveis de severidade da antracnose, reproduzidas em fotocópias coloridas. A severidade foi avaliada por 10 pessoas (A a J) sem experiência na quantificação da doença. Inicialmente, a severidade foi estimada sem o auxílio da escala diagramática e, após sete dias, foi realizada uma primeira avaliação com o auxílio da escala. Visando avaliar a repetibilidade das estimativas com a escala diagramática, sete dias após a primeira avaliação, nova seqüência dos mesmos folíolos foi organizada e uma segunda estimativa visual com auxílio da escala foi efetuada pelos mesmos avaliadores.

A acurácia e a precisão e a reprodutibilidade das estimativas foram determinadas por regressão linear simples, considerando a severidade real como variável independente e a severidade estimada como variável dependente. A acurácia das estimativas de cada avaliador e do conjunto de avaliadores foi determinada pelo teste $t$ aplicado ao intercepto da regressão linear (a), para verificar a hipótese Ho: $a=0$, e ao coeficiente angular da reta $(b)$, para testar a hipótese Ho: $b=1$, ao nível $5 \%$ de probabilidade $(\mathrm{P}=0,05)$. A precisão das estimativas foi determinada pelo coeficiente de determinação da regressão $\left(R^{2}\right)$, pela variância dos erros absolutos (severidade estimada menos severidade real) e pela repetibilidade das estimativas, determinada pela regressão da segunda avaliação em relação à primeira de uma mesma unidade amostral. A reprodutibilidade das estimativas foi determinada pelos valores de $R^{2}$ obtidos de regressões lineares entre as severidades estimadas da mesma unidade amostral por diferentes avaliadores combinados em pares (NUTTER JR \& SCHULTZ, 1995).

O valor máximo de severidade da cercosporiose verificado nos 100 folíolos de caupi foi de $81,8 \%$, enquanto o valor mínimo foi de $0,88 \%$. Considerando esses limites, a escala diagramática elaborada para quantificação da severidade da doença foi representada pelos níveis de 1, 2, 4, 8, 16, 32, 64 e 82\% de área foliar lesionada (Figura 1). Na primeira etapa de validação da escala diagramática, ou seja, sem a sua utilização, os valores do intercepto de $70 \%$ dos avaliadores diferiram significativamente de zero para as retas de regressão entre a severidade real e a estimada, com valor médio de 7,71 (Tabela 1). Com a utilização da escala, os valores do intercepto de $70 \%$ dos avaliadores na primeira avaliação e $60 \%$ na segunda avaliação se mantiveram significativamente diferentes de zero, mas com valores (3,86 e 4,21, respectivamente) inferiores aos constatados sem a utilização da escala. Em todas as situações, os avaliadores superestimaram a severidade da cercosporiose. Os valores do coeficiente angular da reta de 50\% dos avaliadores diferiram significativamente de 1 sem a utilização da escala diagramática, enquanto com a utilização da escala houve redução para 30\% na primeira avaliação e 20\% na segunda avaliação (Tabela 1).

A análise da precisão das estimativas visuais da severidade sem a utilização da escala diagramática justificou de 76 a $86 \%$ da variação $\left(R^{2}\right)$ na mensuração eletrônica da doença, com média de 82\% (Tabela 1). Com a utilização da escala, na primeira avaliação, as estimativas visuais explicaram 91 a 94\% da variação na mensuração eletrônica, com média de 93\%, enquanto na segunda avaliação as estimativas visuais explicaram 91 a 96\% da variação, com média de $94 \%$. A maioria dos avaliadores obteve excelente repetibilidade nas 


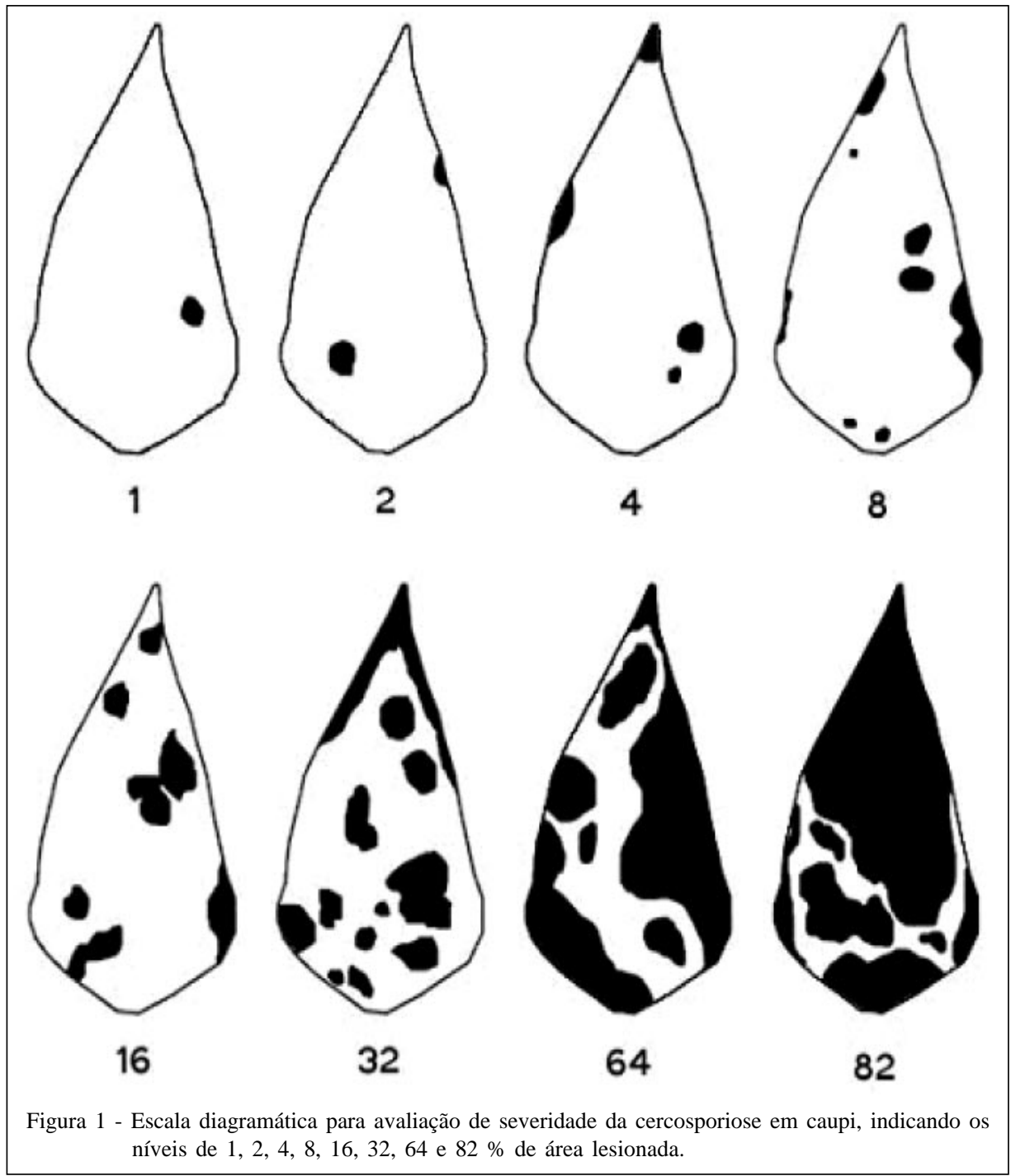

estimativas com a escala diagramática, pois a quantidade média de variação na primeira avaliação explicada pela segunda avaliação foi de $94 \%$. A melhoria da precisão das estimativas na segunda avaliação com a escala em relação à primeira indica que, além da utilização da escala, a familiarização dos avaliadores com a doença e o treinamento destes pode melhorar a acurácia e a precisão das estimativas (NUTTER Jr \& SCHULTZ, 1995).

Houve redução nos erros absolutos para as estimativas com o auxílio da escala diagramática, quando comparada com a distribuição dos resíduos das estimativas obtidas sem a escala. Nas duas avaliações com o auxílio da escala, os resíduos concentraram-se na faixa de $10 \%$, considerados aceitáveis em estudos de avaliação de escalas diagramáticas e que podem ser reduzidos com o treinamento dos avaliadores (NUTTER JR \& SCHULTZ, 1995). Por outro lado, sem a utilização da escala, os erros concentraram-se na faixa de $30 \%$, sendo considerados elevados.

Sem a utilização da escala diagramática, em somente $2,2 \%$ dos casos, a reprodutibilidade das estimativas da severidade da doença foi $\geq 90 \%$, enquanto com o uso da escala diagramática esse nível foi atingido em $68,9 \%$ dos casos na primeira avaliação e $95,6 \%$ dos casos na segunda.

A escala diagramática proposta para quantificação da severidade da cercosporiose em caupi propiciou melhoria significativa nos níveis de acurácia, precisão e reprodutibilidade das estimativas, podendo ser utilizada em estudos epidemiológicos e de comparação de medidas de controle da doença. 
Tabela 1 - Estimativa do intercepto $(a)$, do coeficiente angular da reta $(b)$ e do coeficiente de determinação $\left(R^{2}\right)$ de equações de regressão linear simples relacionando estimativas visuais da cercosporiose do caupi efetuadas por 10 avaliadores, sem e com o auxílio da escala diagramática, à severidade real determinada eletronicamente com auxílio do programa Assess ${ }^{\circledR}$.

\begin{tabular}{|c|c|c|c|c|c|c|c|c|c|}
\hline \multirow{3}{*}{ Avaliador } & \multicolumn{3}{|c|}{--------------Sem escala-------------- } & \multicolumn{6}{|c|}{ 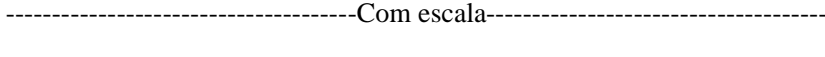 } \\
\hline & \multirow[b]{2}{*}{$a$} & \multirow[b]{2}{*}{$b$} & \multirow[b]{2}{*}{$R^{2}$} & \multicolumn{3}{|c|}{ 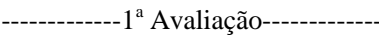 } & \multicolumn{3}{|c|}{ 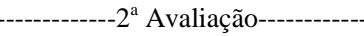 } \\
\hline & & & & $a$ & $b$ & $R^{2}$ & $a$ & $b$ & $R^{2}$ \\
\hline A & $18,66^{*}$ & 1,15 & 0,76 & $4,53 *$ & 0,90 & 0,91 & $5,26 *$ & $0,86^{*}$ & 0,91 \\
\hline B & $12,61^{*}$ & $0,83^{*}$ & 0,82 & $9,93^{*}$ & $0,75^{*}$ & 0,92 & $6,86^{*}$ & $0,87 *$ & 0,93 \\
\hline $\mathrm{C}$ & $-1,41$ & 0,99 & 0,86 & $3,94 *$ & 0,93 & 0,94 & 1,06 & 1,02 & 0,95 \\
\hline $\mathrm{D}$ & $4,87^{*}$ & $0,58^{*}$ & 0,80 & $4,34 *$ & $0,89 *$ & 0,94 & 2,84 & 0,90 & 0,95 \\
\hline E & 1,95 & $0,79 *$ & 0,86 & 1,54 & 1,00 & 0,94 & 1,79 & 0,96 & 0,95 \\
\hline $\mathrm{F}$ & $10,48^{*}$ & 0,91 & 0,85 & $5,34 *$ & 0,92 & 0,91 & $6,06^{*}$ & 0,90 & 0,96 \\
\hline G & $6,45^{*}$ & $0,78^{*}$ & 0,82 & $4,64 *$ & 0,95 & 0,93 & 4,33* & 0,91 & 0,93 \\
\hline $\mathrm{H}$ & $10,53 *$ & $0,71^{*}$ & 0,77 & 1,65 & $0,88^{*}$ & 0,93 & $5,77^{*}$ & 0,95 & 0,94 \\
\hline I & 1,61 & 0,93 & 0,82 & $-2,91$ & 1,00 & 0,92 & 0,57 & 1,00 & 0,95 \\
\hline $\mathrm{J}$ & $11,30^{*}$ & 0,94 & 0,83 & $5,60^{*}$ & 0,95 & 0,92 & $7,56^{*}$ & 1,04 & 0,93 \\
\hline Média & 7,71 & 0,86 & 0,82 & 3,86 & 0,92 & 0,93 & 4,21 & 0,94 & 0,94 \\
\hline
\end{tabular}

*Asterisco representa situações em que a hipótese de nulidade ( $a=0$ ou $b=1$ ) foi rejeitada pelo teste $t(\mathrm{P}=0,05)$.

\section{REFERÊNCIAS}

BERGAMIN FILHO, A.; AMORIM, L. Doenças de plantas tropicais: epidemiologia e controle econômico. São Paulo: Agronômica Ceres, 1996. 299p.

COÊLHO, J.B.; COELHO, R.S.B. Fontes de resistência em caupi (Vigna unguiculata (L.) Walp.) a mancha de cercospora (Cercospora cruenta Sacc.). Revista Ômega - Série Agronomia, v.10, n.1, p.59-63, 1997.

EDEMA, R. et al. Influence of season and cropping system on occurrence of cowpea diseases in Uganda. Plant Disease, v.81, n.4, p.465-468, 1997.

HORSFALL, J.G.; COWLING, E.B. Pathometry: the measurement of plant disease. In: HORSFALL, J.G.; COWLING,
E.B. (Ed.). Plant disease: an advanced treatise - how disease develops in populations. New York: Academic, 1978. Cap.2, p.119-136.

PIO-RIBEIRO, G. et al. Doenças do caupi (Vigna unguiculata). In: KIMATI, H. et al. (Ed.). Manual de fitopatologia: doenças das plantas cultivadas. 4.ed. São Paulo: Agronômica Ceres, 2005. Cap.24, p.215-222.

NUTTER Jr, F.W.; SCHULTZ, P.M. Improving the accuracy and precision of disease assessments: selection of methods and use of computer-aided training programs. Canadian Journal of Plant Pathology, v.17, n.1, p.174-184, 1995.

RIOS, G.P.; ZIMMERMANN, F.J.P. Aspectos epidemiológicos e controle da mancha de cercospora em caupi. Pesquisa Agropecuária Brasileira, v.22, n.2, p.275-279, 1987. 